\title{
Expressivity of Coalgebraic Modal Logic: The Limits and Beyond
}

\author{
Lutz Schröder \\ BISS, Department of Computer Science, University of Bremen
}

\begin{abstract}
Modal logic has a good claim to being the logic of choice for describing the reactive behaviour of systems modeled as coalgebras. Logics with modal operators obtained from so-called predicate liftings have been shown to be invariant under behavioral equivalence. Expressivity results stating that, conversely, logically indistinguishable states are behaviorally equivalent depend on the existence of separating sets of predicate liftings for the signature functor at hand. Here, we provide a classification result for predicate liftings which leads to an easy criterion for the existence of such separating sets, and we give simple examples of functors that fail to admit expressive normal or monotone modal logics, respectively, or in fact an expressive (unary) modal logic at all. We then move on to polyadic modal logic, where modal operators may take more than one argument formula. We show that every accessible functor admits an expressive polyadic modal logic. Moreover, expressive polyadic modal logics are, unlike unary modal logics, compositional.
\end{abstract}

\section{Introduction}

Coalgebra has in recent years emerged as an appropriate framework for the treatment of reactive systems in a very general sense [24]; in particular, coalgebra provides a unifying perspective on notions such as coinduction, corecursion, and bisimulation. It has turned out that modal logic is a good candidate for being the basic logic of coalgebra in the same sense as equational logic is the basic logic of algebra. E.g., classes of coalgebras defined by modal axioms can be regarded as the dual of varieties [12,14. Moreover, coalgebraic modal logic as considered in $[9,13,18,20,19,22$ is invariant under behavioral equivalence. Conversely, in [18, 20, 19, sufficient conditions are given for coalgebraic modal logics to be expressive in the sense that logically indistinguishable states are behaviorally equivalent; this is a generalization of the classical result for HennessyMilner logic [8. These results depend on conditions imposed on the signature functor, i.e. the data type in which collections of successor states are organized.

Indeed, coalgebraic logic as introduced by Moss [16], which may be regarded as a somewhat extreme form of modal logic, is expressive for the (very large) class of so-called set-based functors; however, from the point of view of practical application in software specification, coalgebraic logic has the disadvantage of being rather difficult to grasp, as the syntax and the semantics of its formulae involve applications of the signature functor to the language itself and the 
satisfaction relation, respectively. By comparison, modal logic is rather intuitive and thus well suited for specification purposes. E.g., modal logic is used in the specification of object-oriented programs in the specification language CCSL [23] and in 13 and forms a central feature of the algebraic-coalgebraic specification language CoCASL [17.

Coalgebraic modal logic as developed in [18,20 obtains its modal operators from so-called predicate liftings, which transform predicates on $X$ into predicates on $T X$, where $T$ is the signature functor. Predicate liftings generalize the natural relations considered in [19, which may be regarded as constructions that convert coalgebras into Kripke frames. It is shown in [18,20 that the expressivity problem for coalgebraic modal logic reduces to the existence of enough predicate liftings for the given signature functor; no general answer is given to the question of how to actually find such predicate liftings.

Here, we observe that predicate liftings are equivalent to a notion of modality used in [11]; this affords an immediate overview of all possible predicate liftings of a given functor. Moreover, one obtains easy criteria which identify socalled monotone and continuous predicate liftings, respectively. These properties of predicate liftings correspond to the validity of natural axioms in the arising modal logic; in particular, continuity corresponds to normality. It turns out that continuous predicate liftings essentially coincide with natural relations. These classification results are on the one hand helpful in designing good sets of modal operators for expressive modal logics. On the other hand, they can be used to show that certain signature functors fail to admit expressive monotone or normal modal logics, or indeed an expressive modal logic in the sense considered so far at all. Examples of the latter type include certain composite functors, e.g. the double finite powerset functor, but also single-layer datatypes such as nonrepetitive lists. Typical examples of coalgebras that require non-normal modal logics are those involving some sort of weighting on the successor states, e.g. multigraphs or probabilistic automata.

We then introduce an extension of coalgebraic modal logic in which modal operators may be polyadic, i.e. apply to more than one formula. Both unary and polyadic modal operators may be subsumed under the abstract notion of syntax (or language) constructor [4,5]. Polyadic modal logic, while hardly more complicated than unary modal logic, turns out to be expressive for a large class of functors, the so-called accessible functors. Furthermore, we show that polyadic modal logic is compositional in the sense that expressive modal logics can be combined along functor composition; differently put, polyadic modal logic is, unlike unary modal logic, closed under the composition of syntax constructors.

The material is organized as follows. Section 2 gives an overview of coalgebra and modal logic. Expressivity results for modal logic which assume the existence of enough predicate liftings are discussed in Section 3 , in particular, we improve an expressivity result of [20] and give a simplified proof. We then proceed to discuss the classification of predicate liftings in Section 4 . Finally, polyadic modal logic is treated in Section 5. 


\section{Preliminaries: Coalgebra and Modal Logic}

We now briefly recall the paradigm of modelling reactive systems by means of coalgebras, limiting ourselves to the set-valued case, and the use of modal logic to describe reactive behavior.

Definition 1. Let $T:$ Set $\rightarrow$ Set be a functor (all functors will implicitly be set functors from now on). A $T$-coalgebra $A=(X, \xi)$ consists of a set $X$ of states and an evolution map $\xi: X \rightarrow T X$. A morphism $\left(X_{1}, \xi_{1}\right) \rightarrow\left(X_{2}, \xi_{2}\right)$ of $T$-coalgebras is a map $f: X_{1} \rightarrow X_{2}$ such that $\xi_{2} \circ f=T f \circ \xi_{1}$. A $T$-coalgebra $C$ is called final if there exists, for each $T$-coalgebra $A$, a unique morphism $A \rightarrow C$. Two states $x$ and $y$ in $T$-coalgebras $A$ and $B$ are called behaviorally equivalent if there exists a coalgebra $C$ and homomorphisms $f: A \rightarrow C, g: B \rightarrow C$ such that $f(x)=g(y)$.

The general intuition is that the behavior map describes the successor states of a state, organized in a data structure given by $T$. The notion of behavioral equivalence serves to encapsulate the state space: two states are behaviorally equivalent if the observable aspects of the state evolution from the given states are identical. Thus, the reactive behavior of a state is embodied in its behavioral equivalence class. Final coalgebras are behaviorally abstract in the sense that behaviorally equivalent states are equal; the carrier set of a final coalgebra may be thought of as the set of all possible behaviors. By Lambek's Lemma, the evolution map of a final coalgebra is bijective.

Remark 2. Behavioral equivalence as just defined coincides in most cases with bisimilarity, and appears to be the preferable notion in cases where this fails 12 . Coalgebraic modal logic as treated here captures precisely behavioral equivalence.

Example 3. 1. Let $\mathcal{P}_{\omega}$ be the (covariant) finite powerset functor. Then $\mathcal{P}_{\omega^{-}}$ coalgebras are finitely branching graphs, thought of as (unlabeled) transition systems or indeed Kripke frames.

2. Let $T$ be given by $T X=I \rightarrow \mathcal{P}_{\omega}(X)$ (equivalently $T X=\mathcal{P}_{\omega}(I \times X)$ ). Then $T$-coalgebras are labelled transition systems with label set $I$.

3. Let $T$ be given by $T X=I \rightarrow((O \times X)+E)$. Then $T$-coalgebras may be thought of as modelling objects with state set $X$, method set $I$, output set $O$, and exception set $E[13$. Elements of the final $T$-coalgebra are finite or infinite $I$-branching trees with $O$-labelled nodes and $E$-labelled leaves.

4. Let $T=\mathcal{P}_{\omega} \circ \mathcal{P}_{\omega}$. Then $T$-coalgebras may be thought of as transition systems with two levels of non-determinism; i.e. in each step, a set of possible successors is chosen non-deterministically.

5. The finite multiset (or bag) functor $\mathcal{B}_{\mathbb{N}}$ is given as follows. The set $\mathcal{B}_{\mathbb{N}}(X)$ consists of the maps $B: X \rightarrow \mathbb{N}$ with finite support, where $B(x)=n$ is read ' $B$ contains the element $x$ with multiplicity $n$ '. We write elements of $\mathcal{B}_{\mathbb{N}} X$ additively in the form $\sum n_{i} x_{i}$, thus denoting the multiset that contains $x$ with multiplicity $\sum_{x_{j}=x} n_{j}$. For $f: X \rightarrow Y, \mathcal{B}_{\mathbb{N}}(f)\left(\sum n_{i} x_{i}\right)=\sum n_{i} f\left(x_{i}\right)$. 
Coalgebras for $\mathcal{B}_{\mathbb{N}}$ are directed graphs with $\mathbb{N}$-weighted edges, often referred to as multigraphs 6 .

6. A similar functor, denoted $\mathcal{B}_{\mathbb{Z}}$, is given by a slight modification of the multiset functor where we allow elements to have also negative multiplicities, i.e. $\mathcal{B}_{\mathbb{Z}} X$ consists of finite maps $X \rightarrow \mathbb{Z}$, called generalized multisets (this set is also familiar as the free abelian group over $X$ ).

7. Another variation of the multiset functor is the finite distribution functor $D_{\omega}$, where $D_{\omega} X$ is the set of probability distributions on $X$ with finite support. Coalgebras for $D_{\omega}$ are probabilistic transition systems (as yet without inputs).

8. Examples [5] above may be extended by taking into account a notion of input, with input alphabet $I$, as in Example 2] for $T \in\left\{\mathcal{B}_{\mathbb{N}}, \mathcal{B}_{\mathbb{Z}}, D_{\omega}\right\}$, one has functors $S$ and $R$ given by $S X=I \rightarrow T X$ and $R X=T(I \times X)$. These functors are isomorphic for $T \in\left\{\mathcal{B}_{\mathbb{N}}, \mathcal{B}_{\mathbb{Z}}\right\}$ in case $I$ is finite, but not for $T=D_{\omega}$. In the latter case, $S$-coalgebras are reactive probabilistic automata, and $R$-coalgebras are generative probabilistic automata [2] (more precisely, one would usually allow for terminal states by additionally introducing the constant functor 1 as a summand), the difference being that generative probabilistic automata assign probabilities also to inputs.

All of the above examples fall into the following class of functors:

Definition 4. A functor $T$ is called $\kappa$-accessible, where $\kappa$ is a regular cardinal, if $T$ preserves $\kappa$-directed colimits.

Accessible functors have final coalgebras [1,21].

Example 5. Parametrized algebraic datatypes defined in terms of constructors and equations (i.e. quotients of term algebra functors) are $\kappa$-accessible functors if all constructors have arity less than $\kappa$. E.g., the multiset functors $\mathcal{B}_{\mathbb{N}}$ and $\mathcal{B}_{\mathbb{Z}}$ are $\omega$-accessible. The finite distribution functor $D_{\omega}$ is $\omega$-accessible. For each regular cardinal $\kappa$, the functor $\mathcal{P}_{\kappa}$ given by $\mathcal{P}_{\kappa}(X)=\{A \subset X|| A \mid<\kappa\}$ is $\kappa$-accessible. The class of $\kappa$-accessible functors is closed under composition; e.g. $\mathcal{P}_{\omega} \circ \mathcal{P}_{\omega}$ is $\omega$-accessible.

Remark 6. In all results presented below, $\kappa$-accessibility can in fact be replaced by preservation of $\kappa$-directed unions. We have refrained from making this explicit in all statements, in favor of using standard terminology.

In order to specify requirements on coalgebraic systems in a way that guarantees invariance under behavioral equivalence, coalgebraic logic for so-called Kripke polynomial functors has been introduced (with variations in the syntax) e.g. in $9,13,22$. These results have been generalized in 18, 19, 20, where coalgebraic modal logics are defined on the basis of given natural relations and predicate liftings for the signature, respectively, as follows.

Definition 7. A predicate lifting for a functor $T$ is a natural transformation

$$
\lambda: 2^{-} \rightarrow 2^{T}
$$


where 2- denotes the contravariant powerset functor $\operatorname{Set}^{o p} \rightarrow$ Set, with $2^{f}(A)=$ $f^{-1}[A]$. Explicitly, a predicate lifting assigns to each $A \subset X$ a set $\lambda_{X}(A) \subset T X$ such that

$$
T f^{-1}\left[\lambda_{Y}(A)\right]=\lambda_{X}\left(f^{-1}[A]\right)
$$

for all maps $f: X \rightarrow Y$. A predicate lifting $\lambda$ is called monotone if $A \subset B \subset X$ implies $\lambda_{X}(A) \subset \lambda_{X}(B)$, and continuous if $\lambda_{X}$ preserves intersections for each set $X$, i.e. $\lambda_{X}\left(\bigcap_{i \in I} A_{i}\right)=\bigcap_{i \in I} \lambda_{X}\left(A_{i}\right)$.

A predicate lifting $\lambda$ is equivalently described by its transposite $\lambda^{b}: T \rightarrow$ $2^{\left(2^{-}\right)}$, given by $\lambda_{X}^{b}(t)=\left\{A \subset X \mid t \in \lambda_{X}(A)\right\}$. A set $\Lambda$ of predicate liftings for $T$ is called separating if for each set $X$, the source of maps

$$
\left(\lambda_{X}^{b}: T \rightarrow 2^{\left(2^{-}\right)}\right)_{\lambda \in \Lambda}
$$

is jointly injective, in other words: $t \in T X$ is uniquely determined by the set $\left\{(\lambda, A) \in \Lambda \times 2^{X} \mid t \in \lambda_{X}(A)\right\}$; this property is called separation at $X$.

We shall need the following fact proved in [20]:

Proposition 8. A set $\Lambda$ of predicate liftings for a $\kappa$-accessible functor is separating iff separation holds at all sets $X$ such that $|X|<\kappa$.

Definition 9. Let $T$ be a functor. A language for $T$-coalgebras is a set $\mathcal{L}$ of formulae, equipped with a family of satisfaction relations $\models_{(X, \xi)}$ (or just $\models$ ) between states of $T$-coalgebras $(X, \xi)$ and formulae $\phi \in \mathcal{L}$; we define $\llbracket \phi \rrbracket_{(X, \xi)}$ (or just $\llbracket \phi \rrbracket)$ as the set $\left\{x \in X \mid x \models_{(X, \xi)} \phi\right\}$.

States $x$ and $y$ in $T$-coalgebras $A$ and $B$, respectively, are called logically indistinguishable under $\mathcal{L}$ if

$$
x \models \phi \quad \text { iff } \quad y \models \phi
$$

for all $\phi \in \mathcal{L}$. The language $\mathcal{L}$ is called adequate if behaviorally equivalent states are logically indistinguishable, equivalently: the satisfaction of formulae is invariant under $T$-coalgebra morphisms.

Remark 10. One can define a formula $\phi \in \mathcal{L}$ to be valid in a coalgebra $(X, \xi)$ if $x \models \phi$ for all $x \in X$. This makes $\mathcal{L}$ into a logic for coalgebras as defined in 14. If $T$ has a final coalgebra, then adequacy of $\mathcal{L}$ guarantees that classes of coalgebras defined by axioms in $\mathcal{L}$ have final models [14].

Coalgebraic modal logic [18, 20] is a language $\mathcal{L}^{\kappa}(\Lambda)$ for $T$-coalgebras, parametrized by a set $\Lambda$ of predicate liftings for $T$ and a regular cardinal $\kappa$ which serves as a bound for conjunctions: formulae $\phi \in \mathcal{L}^{\kappa}(\Lambda)$ are defined by the grammar

$$
\begin{array}{rlrl}
\phi::= & {[\lambda] \phi} & (\lambda \in \Lambda) \\
\mid \bigwedge_{i \in I} \phi_{i} & (|I|<\kappa) \\
\mid \neg \phi_{0} . &
\end{array}
$$


Disjunctions $\bigvee_{i \in I} \phi_{i}$ for $|I|<\kappa$ are then defined as usual. In the definition of satisfaction, the clauses for conjunction and negation are as expected; the clause for the modal operator $[\lambda]$ is

$$
x \models_{(X, \xi)}[\lambda] \phi \Longleftrightarrow \xi(x) \in \lambda_{X} \llbracket \phi \rrbracket_{(X, \xi)} .
$$

The naturality equation for predicate liftings is easily seen to be precisely the condition that is needed in order to ensure adequacy of $\mathcal{L}^{\kappa}(\Lambda)$ 20. The converse of this statement, i.e. the question under which conditions $\mathcal{L}^{\kappa}(\Lambda)$ and related logics are expressive, is the main subject of this paper.

The construction of $\mathcal{L}^{\kappa}(\Lambda)$ presupposes that a suitable set of predicate liftings for $T$ is already given. We will discuss in Section 4 how predicate liftings may be obtained and classified in general.

Definition 11. A natural relation for $T$ is a natural transformation $\mu: T \rightarrow \mathcal{P}$.

Thus, for a natural relation $\mu$, composition with $\mu_{X}$ converts $T$-coalgebras on $X$ into Kripke frames. A natural relation $\mu$ induces (transposites of) predicate liftings by composing with transposites of predicate liftings for $\mathcal{P}$ :

$$
T \rightarrow \mathcal{P} \rightarrow 2^{\left(2^{-}\right)} .
$$

In fact, it suffices to consider the composite $\left(\lambda^{\forall}\right)^{b} \circ \mu$, where $\lambda_{X}^{\forall}(A)=\{B \in$ $\mathcal{P}(X) \mid B \subset A\}$; this will be treated in more detail in Section 4

\section{Expressivity of Coalgebraic Modal Logic}

We now turn to the question of when coalgebraic modal logic is strong enough to distinguish behaviorally inequivalent states.

Definition 12. A language $\mathcal{L}$ for $T$-coalgebras is called expressive if logical indistinguishability under $\mathcal{L}$ implies behavioral equivalence.

It is shown in [18 that, for $T \kappa$-accessible and $\Lambda$ separating, $\mathcal{L}^{\sigma}(\Lambda)$ is expressive for 'sufficiently large' $\sigma$ in the stronger sense that behavioral equivalence classes are characterized by single formulae. Moreover, it is shown in 20] that under the same assumptions, $\mathcal{L}^{\kappa}(\Lambda)$ is expressive in the sense defined above, provided that either $\alpha<\kappa$ implies $2^{\alpha}<\kappa$ (i.e. $\kappa=\omega$ or $\kappa$ strongly inaccessible) or the predicate liftings in $\Lambda$ are continuous. These restrictions are quite strong: even the mere existence of strongly inaccessible cardinals is unprovable in ZFC, and the next section will show that continuous predicate liftings are in fact just natural relations. The proofs in [18,20] are by terminal sequence induction. Note that the subtle-appearing difference between the two expressiveness results is in fact rather substantial. E.g. in the case of labelled transition systems (Example 312), the first result concerns a modal logic with countably infinitary conjunction, while the second result asserts the expressivity of standard Hennessy-Milner logic with finitary conjunction.

We now give an improved version of the second result, in which the additional assumptions on $\kappa$ and $\Lambda$, respectively, are dropped. 
Theorem 13. Let $T$ be $\kappa$-accessible and let $\Lambda$ be a separating set of predicate liftings. Then $\mathcal{L}^{\kappa}(\Lambda)$ is expressive.

Proof. (Sketch) One has to show that a given $T$-coalgebra $(X, \xi)$ can be quotiented by the logical indistinguishability relation $R$. This leads to a welldefinedness problem, which may be solved using separation under $\Lambda$ and the fact that on $Z \subset X$ with $|Z|<\kappa$, sets that are closed under $R$ can be described by a $\mathcal{L}^{\kappa}(\Lambda)$-formula.

The above expressivity result has a partial converse:

Theorem 14. If $T$ is $\kappa$-accessible and the final $T$-coalgebra $(Z, \zeta)$ satisfies $|Z| \geq$ $\kappa$, then expressivity of $\mathcal{L}^{\sigma}(\Lambda)$ for some $\sigma$ implies that $\Lambda$ is separating.

Example 15. The assumption $|Z| \geq \kappa$ in the above theorem is essential. As a simple example where $|Z|<\kappa$, consider the non-empty finite powerset functor $\mathcal{P}_{\omega}^{*}$ (i.e. $\mathcal{P}_{\omega}^{*}(X)=\left\{A \in \mathcal{P}_{\omega}(X) \mid A \neq \emptyset\right\}$ ). The final coalgebra for this functor is a singleton. Thus, all states are behaviorally equivalent, so that any logic is expressive for $T$, including e.g. $\mathcal{L}^{\omega}(\emptyset)$; of course, the empty set of predicate liftings is not separating. The same holds for the functor $\mathcal{P}_{\omega}^{*} \circ \mathcal{P}_{\omega}^{*}$, which as we shall see below does not admit a separating set of predicate liftings at all.

\section{Classification of Predicate Liftings}

As indicated above, no general method has been given so far to actually construct predicate liftings for a given functor. The following simple fact (essentially just the Yoneda Lemma for the functor $2^{T}:$ Set $^{o p} \rightarrow$ Set) gives immediate access to all predicate liftings that a functor admits.

Proposition 16. Predicate liftings for $T$ are in one-to-one correspondence with subsets of $T 2$, where $2=\{\top, \perp\}$. The correspondence takes a predicate lifting $\lambda$ to $\lambda_{2}(\{\top\}) \subset T 2$ and, conversely, $C \subset T 2$ to the predicate lifting $\lambda^{C}$ defined by

$$
\lambda_{X}^{C}(A)=\left(T \chi_{A}\right)^{-1}[C]
$$

for $A \subset X$, where $\chi_{A}: X \rightarrow 2$ is the characteristic function of $A$.

Remark 17. Subsets of $T 2$, i.e. $T$-algebras on 2 , have appeared as modalities in [1]. Proposition [16] establishes that this notion of modality and the one induced by predicate liftings are equivalent.

We shall thus freely apply terminology introduced so far for predicate liftings to subsets of $T 2$ as well. E.g. we say that a set of subsets of $T 2$ is separating if the associated set of predicate liftings is separating, etc. Proposition [16] leads to a criterion for the existence of separating sets of predicate liftings, and hence of expressive modal logics. 
Corollary 18. A functor $T$ has a separating set of predicate liftings iff the source

$$
\mathcal{S}_{X}=(T f: T X \rightarrow T 2)_{f: X \rightarrow 2}
$$

is jointly injective at each set $X$. If $T$ is $\kappa$-accessible, then joint injectivity of $\mathcal{S}_{X}$ for $|X|<\kappa$ is sufficient.

Example 19. 1. The (finite) powerset functor has, by Proposition 16, precisely 16 predicate liftings, generated as boolean combinations of the predicate liftings $\lambda^{\forall}$ and $\lambda^{\exists}$ corresponding to $\{\emptyset,\{\top\}\},\{\{\top\},\{\top, \perp\}\} \subset \mathcal{P} 2$, respectively; i.e. $\lambda^{\forall}(A)=\{B \mid B \subset A\}$ and $\lambda^{\exists}(A)=\{B \mid B \cap A \neq \emptyset\}$. The predicate lifting $\lambda^{\forall}$ is continuous; the set $\left\{\lambda^{\forall}\right\}$ is separating. The modalities induced by $\lambda^{\forall}$ and $\lambda^{\exists}$ are the usual operators of modal logic.

2. A close relative of the functors $\mathcal{P}_{\omega}, \mathcal{B}_{\mathbb{N}}$, and the list functor list is the functor $T$ that takes a set $X$ to the free idempotent monoid (or free band monoid) over $X$. The set $T X$ is obtained as the quotient of list $X$ modulo idempotence, i.e. the equation $x x=x$. (Subsequent quotienting modulo commutativity produces $\mathcal{P}_{\omega}$.) By Corollary 18, $T$ does not admit a separating set of predicate liftings: the elements of $T\{a, b, c\}$ represented by abaca and $a b c a$, respectively, are distinct (see e.g. 25]), but identified under $T f$ for all $f:\{a, b, c\} \rightarrow 2$ (e.g. $T \chi_{\{b, c\}}(a b a c a)=\perp \top \perp \top \perp=\perp \top \perp=\perp \top \top \perp=$ $\left.T \chi_{\{b, c\}}(a b c a)\right)$.

3. Let $T$ be the non-repetitive list functor; i.e. $T X$ is the set of lists over $X$ containing every element of $X$ at most once, and $T f(l)$ is obtained by removing duplicates leftmost first in (list $f)(l)$. By Corollary [18. $T$ does not admit a separating set of predicate liftings, since $a b c, b a c \in T\{a, b, c\}$ are identified under $T f$ for all $f:\{a, b, c\} \rightarrow 2$.

4. The double finite powerset functor $T=\mathcal{P}_{\omega} \circ \mathcal{P}_{\omega}$ does not admit a separating set of predicate liftings. E.g., given a finite set $X$, the set $\{A \subset X|| A \mid \leq 2\}$ is identified with $\mathcal{P}_{\omega}(X)$ under $T f$ for all $f: X \rightarrow 2$. A similar argument works for $\mathcal{P}_{\omega} \circ$ list.

Provided the criterion of Corollary 18 is satisfied, the separation property for a given set of predicate liftings can be checked at the level of subsets of $T 2$ :

Theorem 20. Let $T$ admit a separating set of predicate liftings, and let $\mathfrak{C} \subset$ $\mathcal{P}(T 2)$. The following are equivalent:

(i) $\mathfrak{C}$ is separating

(ii) $\operatorname{cl}(\mathfrak{C})=\left\{(T f)^{-1}[C] \mid C \in \mathfrak{C}, f: 2 \rightarrow 2\right\}$ is separating

(iii) $t \in T 2$ is uniquely determined by the set $\{C \in \operatorname{cl}(\mathfrak{C}) \mid t \in C\}$.

We have seen in Example 19 that accessible functors may fail to admit an expressive (unary) modal logic. We now proceed to investigate the relationship between typical modal axioms and properties of predicate liftings, with a view to giving further separating examples.

Generally, a modal operator $\square$ is called monotone $[3$ if it satisfies the axiom scheme $\square(\phi \wedge \psi) \Longrightarrow \square \phi$, often referred to as axiom $M$. Moreover, $\square$ is $\alpha$-normal 
for a regular cardinal $\alpha$ if it satisfies the axiom scheme $\bigwedge_{i \in I} \square \phi_{i} \Longleftrightarrow \square \bigwedge_{i \in I} \phi_{i}$ for $|I|<\alpha$. Note that $\omega$-normality is semantically equivalent to the usual notion of normality for modal operators, i.e. the necessitation rule ('conclude $\square \phi$ from $\left.\phi^{\prime}\right)$ and the $K$-axiom $\square(\phi \Rightarrow \psi) \Rightarrow(\square \phi \Rightarrow \square \psi)$ (equivalently: $\square \phi \Rightarrow(\square \psi \Rightarrow$ $\square(\phi \wedge \psi)))$. In a nutshell, monotone predicate liftings correspond to monotone modal logic, and continuous predicate liftings correspond to normal modal logic:

Theorem 21. Let $T$ be a functor, and let $\lambda$ be a predicate lifting for $T$. If $\lambda$ is monotone then $[\lambda]$ is monotone. Conversely, if $T$ is $\kappa$-accessible, $T$ admits a separating set of predicate liftings, the final $T$-coalgebra $(Z, \zeta)$ satisfies $|Z| \geq \kappa$, and $[\lambda]$ is monotone, then $\lambda$ is monotone.

Definition 22. For a regular cardinal $\beta$, we define $\bar{\beta}$ to be the smallest cardinal such that $2^{\alpha}<\bar{\beta}$ for all $\alpha<\beta$.

E.g. $\bar{\omega}=\omega$, and $\bar{\beta}=\beta$ for $\beta$ strongly inaccessible. Under GCH, $\bar{\beta}$ is either $\beta$ or $2^{\beta}$.

Proposition 23. If $T$ is $\kappa$-accessible, then a predicate lifting $\lambda$ for $T$ is continuous iff $\lambda_{X}$ preserves intersections for $|X|<\kappa$ iff $\lambda_{X}$ preserves intersections of less than $\bar{\kappa}$ sets.

Corollary 24. If $T$ is $\omega$-accessible, then a predicate lifting $\lambda$ for $T$ is continuous iff $\lambda_{X}(X)=T X$ for all $X$ and $\lambda_{X}(A \cap B)=\lambda_{X}(A) \cap \lambda_{X}(B)$ for all $A, B \subset X$.

Theorem 25. Let $T$ be a functor, and let $\lambda$ be a predicate lifting for $T$. If $\lambda$ is continuous, then the modal operator $[\lambda]$ is $\alpha$-normal for all regular cardinals $\alpha$. Conversely, if $T$ is $\kappa$-accessible, $T$ admits a separating set of predicate liftings, the final $T$-coalgebra $(Z, \zeta)$ has $|Z| \geq \kappa$, and $[\lambda]$ is $\bar{\kappa}$-normal, then $\lambda$ is continuous.

As announced above, continuous predicate liftings 'are' natural relations:

Theorem 26. A predicate lifting $\lambda$ for $T$ is continuous iff its transposite $\lambda^{b}$ is of the form $\left(\lambda^{\forall}\right)^{b} \circ \mu$ (cf. Example 19) for some natural relation $\mu: T \rightarrow \mathcal{P}$.

(A dual result holds for predicate liftings with transposites of the form $\left(\lambda^{\exists}\right)^{b} \circ \mu$; in pointwise form, this appears essentially already in [10].)

Corollary 27. A functor admits a separating set of natural relations iff it admits a separating set of continuous predicate liftings.

The slogan is thus that normal coalgebraic modal logic is the logic of natural relations.

We now give criteria for the monotonicity and continuity of predicate liftings at the level of subsets of $T 2$. This will enable us to give examples separating modal logic, monotone modal logic, and normal modal logic w.r.t. expressive strength. 
Proposition 28. Let 3 denote the set $\{\perp, *, \top\}$. A subset $C \subset T 2$ is monotone iff for each $t \in T 3, T \chi_{\{\top\}}(t) \in C$ implies $T \chi_{\{*, T\}}(t) \in C$.

Remark 29. If $T$ is a parametrized algebraic datatype (Example 5), then the condition of the above proposition informally states that $C$, which then consists of equivalence classes of terms in the variables $T$ and $\perp$, is closed under replacing any number of occurrences of $\perp$ in a term by $T$.

Proposition 30. Let $T$ be $\omega$-accessible. A monotone subset $C \subset T 2$ is continuous iff, for each $t \in T\{\perp, a, b, \top\}, T \chi_{\{\top\}}(t) \in C$ whenever $T \chi_{\{a, \top\}}(t) \in C$ and $T \chi_{\{b, T\}}(t) \in C$.

Remark 31. If $T$ is a parametrized algebraic datatype, then the condition of the above proposition informally states that if two sets of occurrences of $T$ in a term representing an element of $C \subset T 2$ may separately be replaced by $\perp$, resulting in terms that remain in $C$, then replacing all occurrences in the two sets simultaneously also yields a term in $C$.

Example 32. 1. For the finite multiset functor $\mathcal{B}_{\mathbb{N}}$ (Example 35 ), $\mathcal{B}_{\mathbb{N}} 2$ consists of elements of the form $n \top+m \perp$. By Remark 29] a subset $C$ of $\mathcal{B}_{\mathbb{N}} 2$ is monotone iff $n \top+(m+k) \perp \in C$ implies $(n+k) \top+m \perp \in C$. A separating set of monotone predicate liftings $\lambda^{k}, k \in \mathbb{N}$, is induced by the subsets of $\mathcal{B}_{\mathbb{N}} 2$ of the form $C_{k}=\{n \top+m \perp \mid m \leq k\}$. The arising modal operators are exactly the modalities $[k]$ of graded modal logic (cf. e.g. 6]). Of course, $[k]$ fails to be normal unless $k=0$.

The functor $\mathcal{B}_{\mathbb{N}}$ does not admit a separating set of continuous predicate liftings, i.e. does not admit an expressive normal modal logic: using Proposition 30 . one can show that all continuous predicate liftings for $\mathcal{B}_{\mathbb{N}}$ besides $\lambda^{0}$ are induced by $\{n \top+m \perp \mid n+m \in A\}$ for some $A \subset \mathbb{N}$.

2. The generalized multiset functor $\mathcal{B}_{\mathbb{Z}}$ (Example 36 ) even fails to admit a separating set of monotone predicate liftings, i.e. does not admit an expressive monotone modal logic: the description of monotone subsets $C \subset \mathcal{B}_{\mathbb{Z}} 2$ is as for $\mathcal{B}_{\mathbb{N}}$ above, but with $k \in \mathbb{Z}$, so that $C=\{n \top+m \perp \mid n+m \in A\}$ for some $A \subset \mathbb{Z}$. A separating set of non-monotone predicate liftings $\lambda^{k}, k \in \mathbb{Z}$, for $\mathcal{B}_{\mathbb{Z}}$ is given by the subsets $C_{k}=\{n \top+m \perp \mid m \leq k\}$.

3. The finite distribution functor $D_{\omega}$ does not admit a separating set of continuous predicate liftings; this is shown in the same way as for $\mathcal{B}_{\mathbb{N}}$. A separating set of monotone predicate liftings is given by the sets $C_{p}=\left\{P \in D_{\omega} 2 \mid\right.$ $P\{\top\} \geq p\}$. These predicate liftings give rise to probabilistic modal operators $[p]$, where $[p] \phi$ reads ' $\phi$ holds in the next step with probability at least $p^{\prime}$ (this modal operator appears in [4]; similar operators are used e.g. in [15]).

4. When the above examples are extended with inputs from a set $I$ as laid out in Example 38, one obtains essentially the same modalities as above, indexed over $a \in I$ in the form $[-]_{a}$. In the case $T=D_{\omega}$, the meaning of $[p]_{a} \phi$ in reactive probabilistic automata is that on input $a, \phi$ holds in the next step with probability at least $p$, and in generative probabilistic automata that with probability at least $p$, the input is $a$ and $\phi$ holds in the next step. 
There is a canonical way to produce predicate liftings which often leads to useful modal operators: one can just apply $T$ to subsets of 2 . In particular, the predicate lifting given by $T\{T\}$ is often important; in fact, this is the principle which is currently used for the definition of modal operators in CoCASL [17].

\section{Polyadic Coalgebraic Modal Logic}

Having seen in the preceding section that accessible functors may fail to admit separating sets of predicate liftings, we now proceed to develop a slightly generalized framework that yields expressive logics for all accessible functors. Essentially, all one has to do is to move on from unary modal operators to polyadic modal operators. Polyadic modal operators for coalgebras rely on the following notion of polyadic predicate lifting.

Definition 33. An $\alpha$-ary predicate lifting for a functor $T$, where $\alpha$ is a cardinal, is a natural transformation

$$
\lambda:\left(2^{-}\right)^{\alpha} \rightarrow 2^{T^{o p}}
$$

A set $\Lambda$ of such polyadic predicate liftings is called $\kappa$-bounded if all predicate lifings in $\Lambda$ have arity properly smaller than $\kappa$ (in particular $\Lambda$ is $\omega$-bounded if all predicate liftings in $\Lambda$ are finitary). Moreover, $\Lambda$ is called separating if the associated source of transposites

$$
\left(\lambda^{b}: T \rightarrow 2^{\left(\left(2^{-}\right)^{\alpha}\right)}\right)_{\lambda \in \Lambda},
$$

formed analogously to the unary case, is injective at each set $X$.

Explicitly, the naturality condition states that, for each map $f: X \rightarrow Y$ and each family $\left(A_{i}\right)_{i \in \alpha}$ of $\alpha$ subsets $A_{i} \subset Y$,

$$
T f^{-1}\left[\lambda_{Y}\left(A_{i}\right)_{i \in \alpha}\right]=\lambda_{X}\left(f^{-1}\left[A_{i}\right]\right)_{i \in \alpha}
$$

The polyadic modal language is then defined as follows.

Definition 34. Let $T$ be a functor, let $\Lambda$ be a set of polyadic predicate liftings for $T$, and let $\kappa$ be a cardinal. The language $\mathcal{L}^{\kappa}(\Lambda)$ is defined as in the unary case (cf. Section 2), except for application of modal operators: an $\alpha$-ary predicate lifting $\lambda \in \Lambda$ gives rise to an $\alpha$-ary modal operator $[\lambda]$, i.e. we have formulae of the form

$$
[\lambda]\left(\phi_{i}\right)_{i \in \alpha}
$$

where $\left(\phi_{i}\right)_{i \in \alpha}$ is a family of formulae in $\mathcal{L}^{\kappa}(\Lambda)$.

The satisfaction relation over a $T$-coalgebra $(X, \xi)$ is given by the generalized clause

$$
x \models[\lambda]\left(\phi_{i}\right)_{i \in \alpha} \quad \text { iff } \quad \xi(x) \in \lambda_{X}\left(\llbracket \phi_{i} \rrbracket\right)_{i \in \alpha} .
$$


It is easy to see that $\mathcal{L}^{\kappa}(\Lambda)$ is adequate. The expressivity results discussed in Section 3 generalize in a straightforward manner (essentially by inspection of the proofs given above and in [18]), i.e. if $T$ is accessible, $\Lambda$ is a separating set of polyadic predicate liftings, and $\sigma$ is 'sufficiently large', then $\mathcal{L}^{\sigma}(\Lambda)$ has characterizing formulae for behavioral equivalence classes, and

Theorem 35. Let $T$ be $\kappa$-accessible and let $\Lambda$ be a separating set of polyadic predicate liftings for $T$. Then $\mathcal{L}^{\kappa}(\Lambda)$ is expressive.

One has the same simple classification result as for unary predicate liftings:

Proposition 36. For $\alpha$ a cardinal, $\alpha$-ary predicate liftings for $T$ are in one-toone correspondence to subsets of $T\left(2^{\alpha}\right)$. The correspondence works by taking a predicate lifting $\lambda$ to $\lambda_{2^{\alpha}}\left(\pi_{i}^{-1}\{\top\}\right)_{i \in \alpha} \subset T\left(2^{\alpha}\right)$, where $\pi_{i}: 2^{\alpha} \rightarrow 2$ is the $i$-th projection, and, conversely, $C \subset T\left(2^{\alpha}\right)$ to the predicate lifting $\lambda^{C}$ defined by

$$
\lambda_{X}^{C}\left(A_{i}\right)_{i \in \alpha}=\left(T\left\langle\chi_{A_{i}}\right\rangle_{i \in \alpha}\right)^{-1}[C]
$$

for $A_{i} \subset X(i \in \alpha)$, where angle brackets are used to denote tupling of functions.

Corollary 37. The functor $T$ admits a separating $\kappa$-bounded set of polyadic predicate liftings iff the the source

$$
\mathcal{S}_{X}=\left(T f: T X \rightarrow T\left(2^{\alpha}\right)\right)_{\alpha<\kappa, f: X \rightarrow 2^{\alpha}}
$$

is injective for each set $X$.

Unlike for unary predicate liftings, we now obtain that all accessible functors admit expressive polyadic modal logics:

Corollary 38. If $T$ is $\kappa$-accessible, then $T$ admits a separating $\kappa$-bounded set of polyadic predicate liftings.

A further issue in coalgebraic modal logic is the modular construction of logics. It has been shown in [18] that separating sets of unary predicate liftings can be propagated along small products of functors, subfunctors (hence along small limits), and small coproducts; by Example 19, however, unary predicate liftings can not be combined along functor composition. Modularity results for expressive languages for accessible functors are proved at a more abstract level in [4, 5, using notions of syntax (or language) constructor and one-step semantics. These results include combinations of syntax constructors and their one-step semantics, respectively, along functor composition.

We now show that separating sets of polyadic predicate liftings can be combined along composition of $\kappa$-accessible functors for arbitrary $\kappa$ (of course, the existence of separating sets for such composites is clear by Corollary (38). The arising modal logic can then be seen to be equivalent, via a simple syntactic transformation, to a multi-sorted modal logic obtained by composing the associated syntax constructors and their one-step semantics according to [4, 5 . 
Thus, polyadic modal logic is essentially closed under the composition operation of 4,5 - i.e. for purposes of the meta-theory, one never has to go beyond the polyadic modal language defined above.

We begin by observing that predicate liftings can be composed:

Proposition and Definition 39. Let $T$ and $S$ be functors, let $\lambda$ be an $\alpha$-ary predicate lifting for $T$, and let $\left(\nu^{i}\right)_{i \in \alpha}$ be a family of predicate liftings for $S$, where $\nu^{i}$ has arity $\beta_{i}$. Then

$$
\left(\lambda \circledast\left(\nu^{i}\right)_{i \in \alpha}\right)_{X}\left(A_{i j}\right)_{i \in \alpha, j \in \beta_{i}}=\lambda_{S X}\left(\nu_{X}^{i}\left(A_{i j}\right)_{j \in \beta_{i}}\right)_{i \in \alpha}
$$

defines a $\sum_{i \in \alpha} \beta_{i}$-ary predicate lifting for $T \circ S$.

Next we note that (possibly infinitary) boolean combinations of polyadic predicate liftings are again predicate liftings:

Proposition and Definition 40. Let $\Lambda$ be a set of polyadic predicate liftings. Then each of the following equations defines a polyadic predicate lifting $\nu$ :

(i) $\nu_{X}\left(A_{i}\right)_{i \in \beta}=\lambda_{X}\left(A_{\Phi(j)}\right)_{j \in \alpha}$, where $\beta$ is a cardinal, $\lambda \in \Lambda$ has arity $\alpha$, and $\Phi$ is a $\operatorname{map} \alpha \rightarrow \beta$;

(ii) $\nu_{X}\left(A_{i}\right)_{i \in \alpha}=T X-\lambda_{X}\left(A_{i}\right)_{i \in \alpha}$, where $\lambda \in \Lambda$ has arity $\alpha$;

(iii) $\nu_{X}\left(A_{i}\right)_{i \in \alpha}=\bigcap_{j \in \gamma} \lambda_{X}^{j}\left(A_{i}\right)_{i \in \alpha}$, where $\gamma$ is a cardinal and for each $j, \lambda^{j} \in \Lambda$ has arity $\alpha$.

The closure of $\Lambda$ under these constructions, with (i) and (iii) restricted to $\beta<\kappa$ and $\gamma<\kappa$, respectively, is called the $\kappa$-boolean closure of $\Lambda$, denoted $\operatorname{bcl}_{\kappa}(\Lambda)$. The elements of this set are called $\kappa$-boolean combinations of $\Lambda$.

The announced compositionality result for separating sets of predicate liftings is the following.

Theorem 41. Let $S$ and $T$ be functors, where $T$ is $\kappa$-accessible for a regular cardinal $\kappa$, and let $\Lambda_{S}$ and $\Lambda_{T}$ be $\kappa$-bounded separating sets of predicate liftings for $S$ and $T$, respectively. Then

$\Lambda_{T} \circledast \operatorname{bcl}_{\kappa}\left(\Lambda_{S}\right)=\left\{\lambda \circledast\left(\nu^{i}\right)_{i \in \alpha} \mid \alpha\right.$ cardinal, $\lambda \in \Lambda_{T} \alpha$-ary, $\nu^{i} \in \operatorname{bcl}_{\kappa}\left(\Lambda_{S}\right)$ for all $\left.i\right\}$ is a $\kappa$-bounded separating set of predicate liftings for $T \circ S$.

If, in the notation of the above theorem, $S$ is $\kappa$-accessible, then it follows from Theorem 35 that $\mathcal{L}^{\kappa}\left(\Lambda_{T} \circledast \operatorname{bcl}_{\kappa}\left(\Lambda_{S}\right)\right)$ is an expressive logic for $T \circ S$-coalgebras. Such an expressive logic can also be obtained by the methods of [4,5, i.e. by composing the syntax constructors associated to $\Lambda_{T}$ and $\Lambda_{S}$, along with their one-step semantics. The result is a multi-sorted modal logic where $\Lambda_{T}$-modalities and $\Lambda_{S}$-modalities appear in alternating layers, with $\Lambda_{T}$-modalities in the outermost layer. This logic can easily be seen to be equivalent to $\mathcal{L}^{\kappa}\left(\Lambda_{T} \circledast \operatorname{bcl}_{\kappa}\left(\Lambda_{S}\right)\right)$; in the translation, boolean operators on formulae are turned into boolean operations on predicate liftings, and two layers of modal syntax in $\mathcal{L}^{\kappa}\left(\Lambda_{T}\right)$ and $\mathcal{L}^{\kappa}\left(\Lambda_{S}\right)$, respectively, are combined into one layer of modal syntax in $\mathcal{L}^{\kappa}\left(\Lambda_{T} \circledast \operatorname{bcl}{ }_{\kappa}\left(\Lambda_{S}\right)\right)$. E.g., if $\lambda \in \Lambda_{T}$ is $\alpha$-ary and $\nu_{i} \in \Lambda_{S}$ for all $i$, then the multi-sorted formula $[\lambda]\left[\nu^{i}\right]\left(\phi_{i j}\right)$ becomes the formula $\left[\lambda \circledast\left(\nu^{i}\right)\right]\left(\phi_{i j}\right)$ of $\mathcal{L}^{\kappa}\left(\Lambda_{T} \circledast \operatorname{bcl}_{\kappa}\left(\Lambda_{S}\right)\right)$. In other words, composites of polyadic modal logics in the sense of [4, 5] can always be flattened into a polyadic modal logic. 


\section{Conclusion}

We have studied expressivity issues in the modal logic of coalgebras based on the notion of predicate lifting, following [18, 20]. In [20], an expressivity result for coalgebraic modal logic has been proved under the assumption that the signature functor admits a separating set of predicate liftings. We have improved this result by dropping restrictions on the accessibility degree of the signature functor. Moreover, we have given a simple classification of predicate liftings which has lead to a necessary and sufficient criterion for the existence of separating sets of predicate liftings, and by means of this criterion we have identified examples of functors that fail to admit an expressive unary modal logic.

We have also related monotonicity and continuity of predicate liftings to monotonicity and normality, respectively, of the induced modal operators. The above-mentioned classification of predicate liftings has then allowed us to give examples separating the coalgebraic expressiveness of modal logic, monotone modal logic, and normal modal logic. Furthermore, we have identified normal modal logic as the modal logic of natural relations as introduced in [19. Since natural relations convert coalgebras into Kripke frames, the latter result lends precision to the claim that normal modal logics describe exactly Kripke frames. More generally, reversing the original viewpoint that modal logic serves as a specification language for coalgebras, our results show that coalgebra constitutes a good semantic framework also for non-normal and even non-monotone modal systems (for non-normal systems cf. also [7]).

Finally, we have proposed to generalize coalgebraic modal logic to include polyadic modal operators based on polyadic predicate liftings. We have shown that all accessible functors admit an expressive polyadic modal logic. Moreover, we have proved a compositionality result stating essentially that polyadic modal logic is stable under the composition of languages described in [5].

Future work will include the exploitation of these results in the practical specification of reactive systems. In particular, modal operators specified in terms of our classification result will be integrated into the design of COCASL.

Acknowledgements. The author wishes to thank Till Mossakowski, Markus Roggenbach, and Horst Reichel for collaboration on CoCASL, and Alexander Kurz, Bartek Klin, and the anonymous referees for useful suggestions for improvement.

\section{References}

[1] M. Barr, Terminal coalgebras in well-founded set theory, Theoret. Comput. Sci. 114 (1993), 299-315.

[2] F. Bartels, A. Sokolova, and E. de Vink, A hierarchy of probabilistic system types, Coalgebraic Methods in Computer Science, ENTCS, vol. 82, Elsevier, 2003.

[3] B. Chellas, Modal logic, Cambridge, 1980.

[4] C. Cîrstea, A compositional approach to defining logics for coalgebras, Theoret. Comput. Sci. 327 (2004), 45-69. 
[5] C. Cîrstea and D. Pattinson, Modular construction of modal logics, Concurrency Theory, LNCS, vol. 3170, Springer, 2004, pp. 258-275.

[6] G. D'Agostino and A. Visser, Finality regained: A coalgebraic study of Scott-sets and multisets, Arch. Math. Logic 41 (2002), 267-298.

[7] H. H. Hansen and C. Kupke, A coalgebraic perspective on monotone modal logic, Coalgebraic Methods in Computer Science (J. Adámek and S. Milius, eds.), ENTCS, vol. 106, Elsevier, 2004, pp. 121-143.

[8] M. Hennessy and R. Milner, Algebraic laws for non-determinism and concurrency, J. ACM 32 (1985), 137-161.

[9] B. Jacobs, Towards a duality result in the modal logic of coalgebras, Coalgebraic Methods in Computer Science, ENTCS, vol. 33, Elsevier, 2000.

[10] B. Jónnson and A. Tarski, Boolean algebras with operators I, Amer. J. Math. 73 (1951), 891-939.

[11] B. Klin, A coalgebraic approach to process equivalence and a coinduction principle for traces, Coalgebraic Methods in Computer Science, ENTCS, vol. 106, Elsevier, 2004, pp. 201-218.

[12] A. Kurz, Logics for coalgebras and applications to computer science, Ph.D. thesis, Universität München, 2000.

[13] _ Specifying coalgebras with modal logic, Theoret. Comput. Sci. 260 (2001), $119-138$.

[14] Logics admitting final semantics, Foundations of Software Science and Computation Structures, LNCS, vol. 2303, Springer, 2002, pp. 238-249.

[15] K. Larsen and A. Skou, Bisimulation through probabilistic testing, Inform. Comput. 94 (1991), 1-28.

[16] L. Moss, Coalgebraic logic, Ann. Pure Appl. Logic 96 (1999), 277-317.

[17] T. Mossakowski, L. Schröder, M. Roggenbach, and H. Reichel, Algebraic-coalgebraic specification in CoCASL, J. Logic Algebraic Programming, to appear.

[18] D. Pattinson, Expressivity results in the modal logic of coalgebras, Ph.D. thesis, Universität München, 2001.

[19] Semantical principles in the modal logic of coalgebras, Symposium on Theoretical Aspects of Computer Science, LNCS, vol. 2010, Springer, 2001, pp. 514 526 .

[20] _ Expressive logics for coalgebras via terminal sequence induction, Notre Dame J. Formal Logic 45 (2004), 19-33.

[21] J. Power and H. Watanabe, An axiomatics for categories of coalgebras, Coalgebraic Methods in Computer Science, ENTCS, vol. 11, Elsevier, 2000.

[22] M. Rößiger, Coalgebras and modal logic, Coalgebraic Methods in Computer Science, ENTCS, vol. 33, Elsevier, 2000.

[23] J. Rothe, H. Tews, and B. Jacobs, The Coalgebraic Class Specification Language CCSL, J. Universal Comput. Sci. 7 (2001), 175-193.

[24] J. Rutten, Universal coalgebra: A theory of systems, Theoret. Comput. Sci. 249 (2000), 3-80.

[25] J. Siekmann and P. Szabo, A noetherian and confluent rewrite system for idempotent semigroups, Semigroup Forum 25 (1982), 83-110. 